\title{
Assessment of productivity of management systems of the multiservice networks
}

\author{
Duravkin Evgen, Mohammed Khodayer Hassan Al-Dulaimi, \\ Aymen Mohammed Khodayer Al-Dulaimi
}

Kharkiv National University of Radio and Electronics, Kharkiv, Ukraine

\section{Email address:}

duravkin_evgen@mail.ru (E. Duravkin), korov4enko@mail.ru (M. K. H. Al-Dulaimi)

\section{To cite this article:}

Duravkin Evgen, Mohammed Khodayer Hassan Al-Dulaimi, Aymen Mohammed Khodayer Al-Dulaimi. Assessment of Productivity of Management Systems of the Multiservice Networks. International Journal of Intelligent Information Systems.

Vol. 2, No. 6, 2013, pp. 94-99. doi: 10.11648/j.ijiis.20130206.11

\begin{abstract}
This article is devoted to the assessment of different service-oriented architectures and development new web-oriented architecture. The current service-oriented architecture such as SOA, COBRA, MWEB considered in the article, reviewed their main advantages and disadvantages in processing large amounts of data. In the range of article done analysis of the response time of distributed multi-service network for different data streams and proposed a new web-oriented architecture of distributed network management. Proposed architecture allows reducing the search time for applications stream that exceeds the service intensity.
\end{abstract}

Keywords: Architecture, Management, Intensity, Probable and Temporal Graph, Request, Service-Oriented

\section{Introduction}

Now in the sphere of infocommunication systems intensive extension of a range of provided services and implementation of new technologies of data transfer is watched. In this regard, the problems of increasing the effectiveness of control systems in telecommunication networks are becoming more and more relevant. Their purpose is to provide the specified quality of service to users in the provision of services.

Today, there are several architectures implementing the concept of management of telecommunication systems of TMN [1]: SNMP [2], CMIP [2], etc. However, with the development of telecommunications networks and the introduction of new services, these technologies do not allow you to fully solve their problems of management and ensure the specified quality of service. First of all this is due to increased heterogeneity of both hardware and software for the implementation of the introduced new services in telecommunications networks.

CORBA [3], SOA [4], WBEM [5] come to change to the specified technologies.

\section{Main Part}

The architecture on the basis of CORBA became one of options of implementation of architecture of distributed control by a network in the concept of TMN. Distributed management framework of info-communication network based on CORBA is presented in Fig.1.

The introduction of technology controls CORBA, based on the idea of open distributed control allows flexibility provide interaction between geographically distributed control system components [6].

It should be noted the focus of these technology on software-implemented components of a distributed control system, that narrows its range of applications. One of the main drawbacks of this technology is that with increase in number of interacting objects (the list of provided services) complexity of implementation of IIOP sharply increases.

WBEM technology involves creation of an open environment for the administration tools that will allow services to interact freely with each other. In this technology is using maximum of existing technologies and standards [3].

WBEM consists of three main components (Figure 3.1) [66]:

1. CommonInformationModel (CIM), which provides a common format, language and methodology for data collection and management.

2. HmlCIM Specification, which defines XML elements, as described in the document type definition (DocumentTypeDefinition, DTD), which is used to represent the CIM 
classes and examples.

3. Specification HTTP over CIM, CIM that defines the transformation operations in HTTP, which allows for interaction with CIM and technologies that support WBEM.(See Fig 3.)

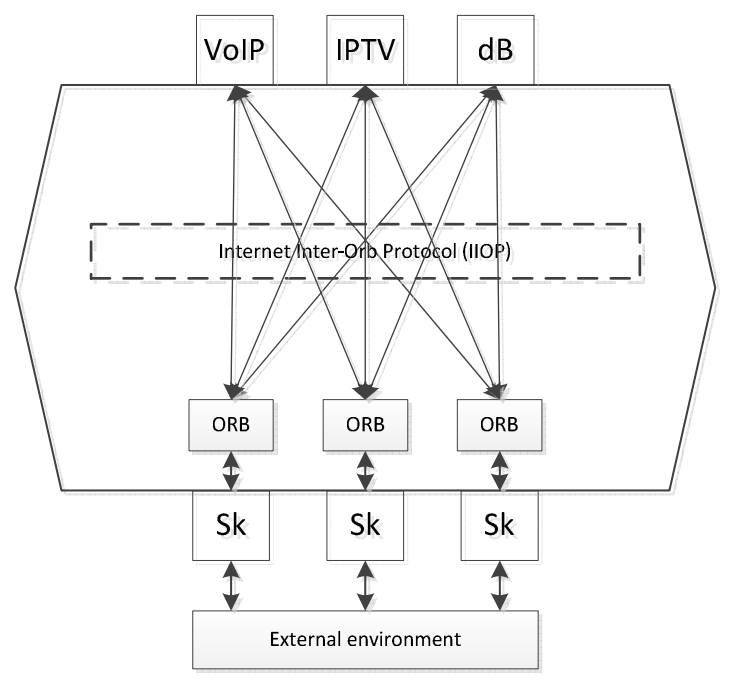

Figure 1. Network structure on the basis of CORBA

The alternative management technology of service-oriented architecture is a Service Oriented Architecture $[4,7]$ (SOA) (Fig. 2).

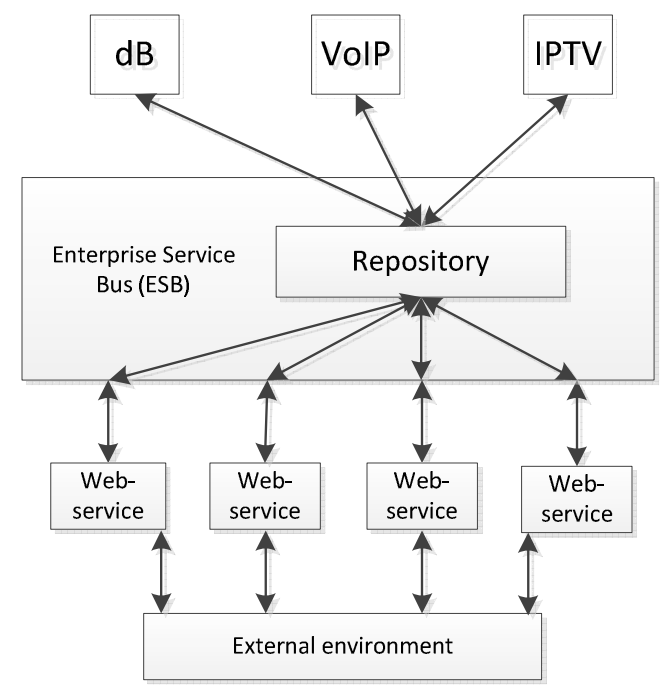

Figure 2. Network structure on the basis of SOA

The main objective of the SOA technology is integration of heterogeneous services provided by a telecommunication network. The technology incorporates the use of a common transport medium (ESB) [6, 7]. Consequently, the performance of the system using this kind of architecture is largely determined by the capacity of the transport medium. In many cases, to achieve high performance of this system is hard enough just because of the heterogeneity of serviced services.

Increase of productivity SOA systems can be achieved in several ways: by improving the characteristics of the ESB;

by combining the provided services to the group and the allocation of individual transport sub-systems for each of the groups.

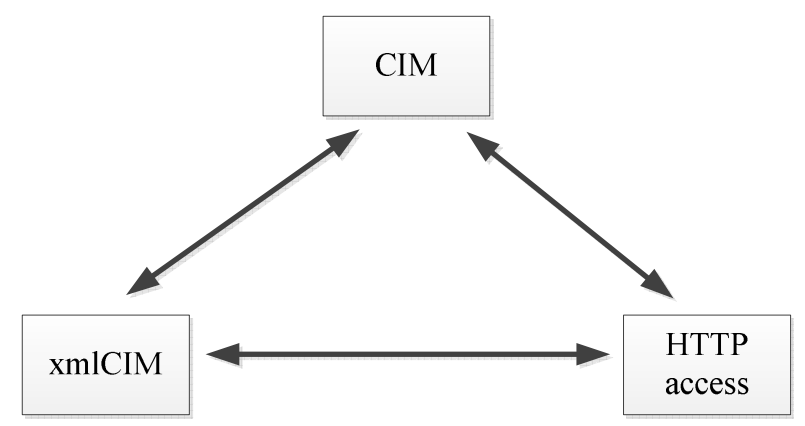

Figure 3. Interaction principle of elements of technology WBEM

When choosing system architecture of control of telecommunication systems it is necessary to carry out comparative an assessment of productivity of management systems a multiservice network implemented on the CORBA, SOA, WBEM technologies.

As the parameter of efficiency of telecommunication systems it is possible to use average time of processing of request a network $\left(T_{I}\right)$ on receiving accidental service for the solution of problems of traffic management. Therefore, the management system shall distribute network resources so as to satisfy the following condition [8]:

$$
T_{I}(\bar{r}) \rightarrow \min .
$$

where $r$ - the distribution vector of network resources.

The considered management system is provided in the form of a state graph, comprises $V$ nodes (possible statuses of system) and $U$ channels (transitions between statuses). In everyone i node arise information flows of requests between $\mathrm{i}$ and $\mathrm{j}$ nodes with intensity $\lambda_{\mathrm{ij}}$. For each flow it is necessary to select network resources to provide required quality of service. In each branch it is necessary to define required band pass range $(\mathrm{C})$ and the necessary capacity of the buffer storage device (B).

For carrying out the analysis of system it is necessary to define network management system model parameters.

Buffer capacity that is allocated to each node for each thread requests will be measured in queries. Performance management system is determined by time the processing of the request $(\mathrm{T})$.

It is necessary to consider that the part of information can be lost. Then the quantity of the processed requests can be provided in the following look:

$$
\operatorname{Ipr}(t)=I_{\text {sum }} \cdot\left(1-P_{l o s}\right),
$$

where $I_{\text {sum }}$ - the number of incoming requests.

$P_{l o s}$ - the probability of losing requests.

Query processing time can be determined according to the formula of queuing theory: 


$$
T_{Q_{i, j}}=\sum_{i, j \in \pi_{i, j}} T_{i, j}=\sum_{i, j \in \pi_{i, j}} \frac{N_{i, j}}{\lambda_{i, j} * P_{l o s i, j}} .
$$

For determination of system effectiveness of control taking into account time of request processing and loss of arriving requests we will use a method of probable and temporal graphs [4]. Therefore, it is necessary to build a probabilistic-time graph that describes the control algo- rithm telecommunication systems using different management architectures. Such graph will allow to evaluate dependence of time of processing of request of the user on receiving accidental (from among supported) services from intensity of arriving requests (network response time).

The structure of distributed network is presented in Fig.4

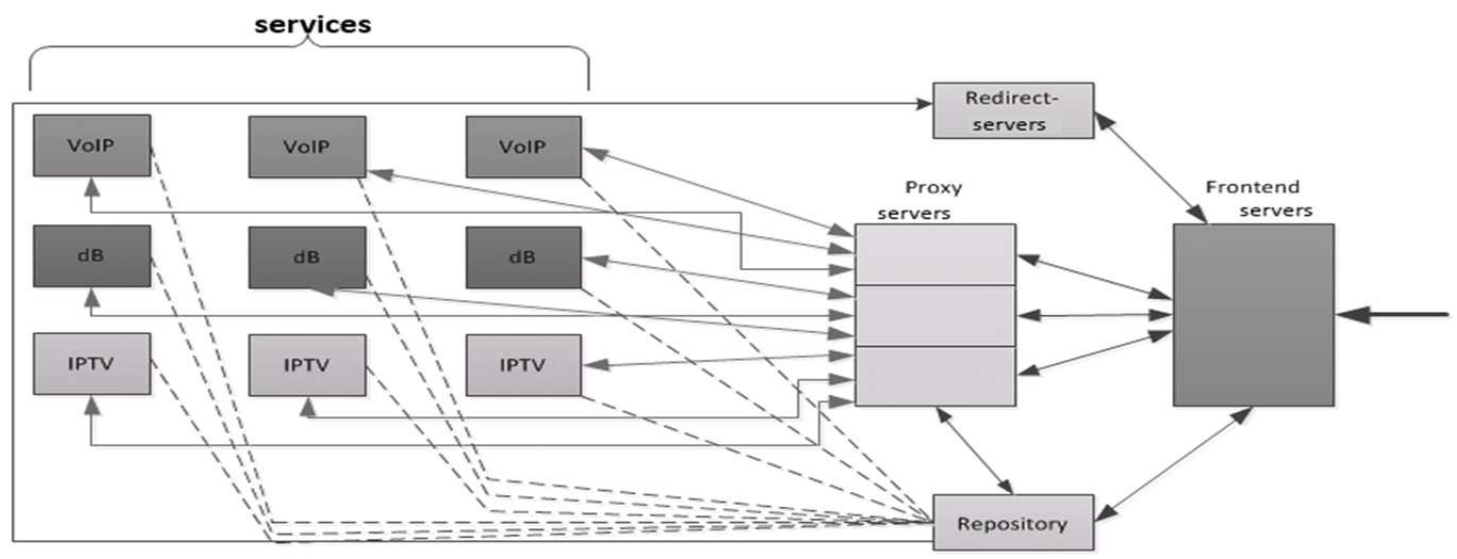

Figure 4. Structure of distributed network on the basis of the offered web-centric architecture

Carry out the comparative analysis of three offered architecture. Probability-time graph describing process of processing of request by distributed system of control on the basis of CORBA, will look like, provided in Fig. 5.

In this figure, indicated by:

$P_{p}$ - probability of error-free delivery of the request;
$P_{f}$ - the probability of finding the requested service on the server;

$P_{n f}$ - the probability that the requested service is not found at the server;

$T_{n}$ - query processing time between nodes $\mathrm{i}$ and $\mathrm{j}$.

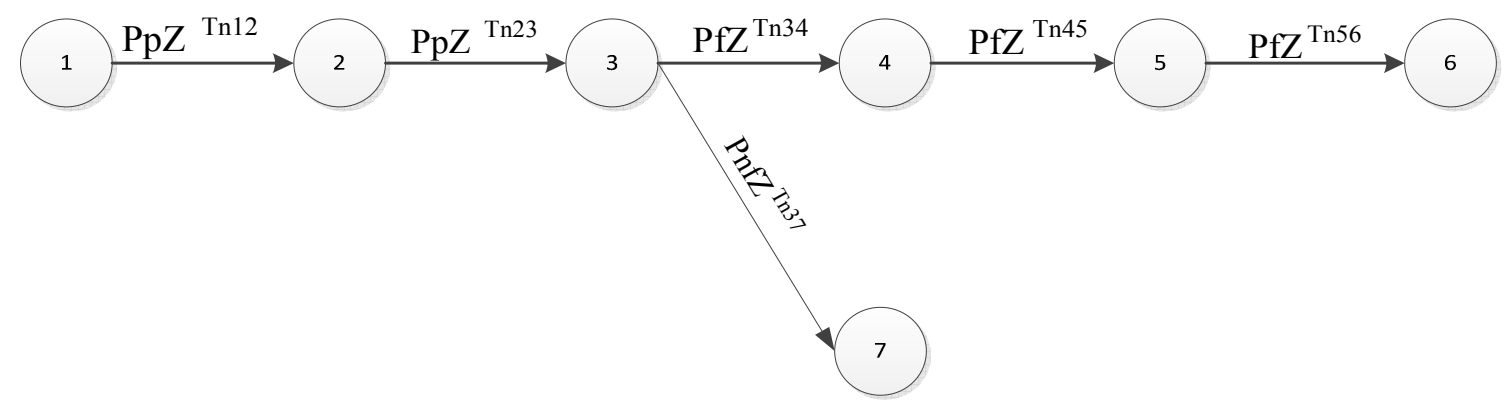

Figure 5. Probability-time graph of the query

The end view of Probability-time graph is presented in Fig.6.

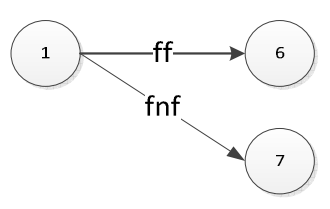

Figure 6. The final form of the converted PTG

The time of servicing a request, provided that the requested service is found:

$$
T_{s r}=\frac{\mathrm{df}_{\mathrm{f}}(\mathrm{z})}{\mathrm{dz}} \mid z=1 .
$$

Where

$$
\mathrm{f}_{\mathrm{f}}(z)=P_{p}^{2} \cdot P_{n f}{ }^{3} \cdot \mathrm{z}^{\mathrm{T}_{12}+T_{23}+T_{34}+T_{45}+T_{56}}
$$

Then the average request processing time, provided that the service is found for a distributed control system based on CORBA is:

$$
\begin{gathered}
T_{s r}=\frac{\mathrm{df}_{\mathrm{f}}(\mathrm{z})}{\mathrm{dz}} \mid(z=1)= \\
=\frac{\mathrm{d}\left(\mathrm{P}_{\mathrm{p}}^{2} \cdot \mathrm{P}_{\mathrm{nf}}^{3} \cdot z^{T 12+T 23+T 34+T 45+T 56}\right)}{\mathrm{dz}}= \\
=P_{p}^{2} \cdot P_{n f}{ }^{3} \cdot\left(T_{12}+T_{23}+T_{34}+T_{45}+T_{56}\right)+ \\
+Z^{T 12+T 23+T 34+T 45+T 56}
\end{gathered}
$$


Probability-time graph (PTG), describing the process of SOA, will have the form shown in Fig. 7. processing the request distributed control system based on

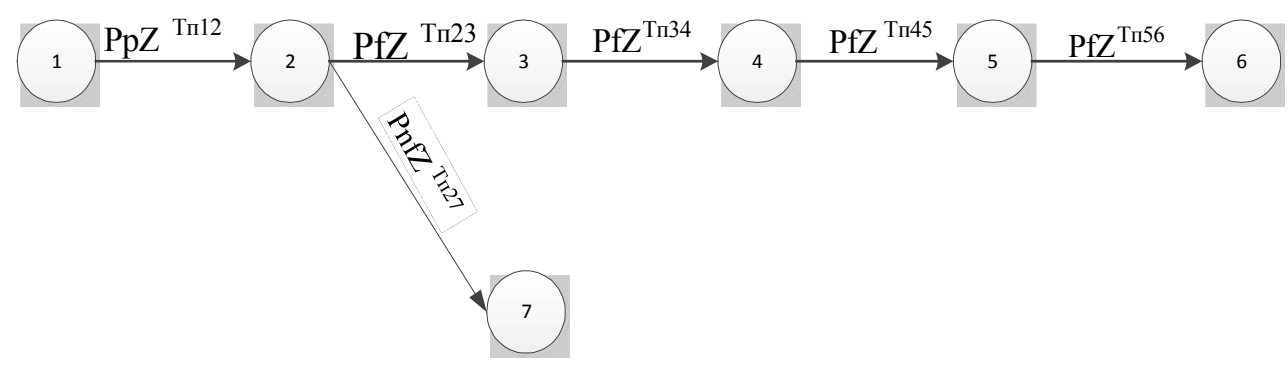

Figure 7. Probable and temporal processing graph of request

On figure variable indicated by:

$P_{p}$ - probability of error-free delivery of the request;

$P_{f}$ - the probability of finding the requested service on the server;

$P_{n f}$ - the probability that the requested service is not found at the server;

$T_{n}$ - query processing time between nodes $\mathrm{i}$ and $\mathrm{j}$.

The time of servicing a request, provided that the requested service is found (Fig.8):

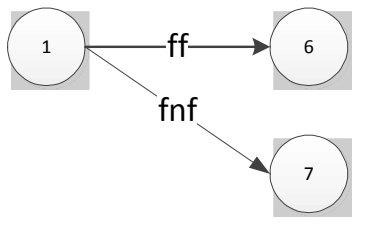

Figure 8. The final form of the converted PTG

$$
T_{s r}=\frac{\mathrm{df}_{\mathrm{f}}(\mathrm{z})}{\mathrm{dz}} \mid z=1
$$

the service is found for a distributed control system based on SOA is:

$$
\begin{aligned}
& T_{s r}=\frac{\mathrm{df}_{\mathrm{f}}(\mathrm{z})}{\mathrm{dz}} \mid(z=1)=P_{p} \cdot P_{f}^{4} \cdot\left(T_{12}+T_{23}+\right. \\
& \left.+T_{34}+T_{45}+T_{56}\right)+Z^{T 12+T 23+T 34+T 45+T 56}
\end{aligned}
$$

Probability-time graph (PTG), describing the process of processing the request distributed control system based on WBEM, will have the form shown in Fig. 9.

Where:

$P_{p}$ - the probability of error-free delivery of the request;

$P_{f}$ - the probability of finding the requested service on the server;

$P_{n f}$ - the probability that the requested service is not found at the server;

$P_{f r}$ - the probability of finding the requested service on a remote server;

$T_{n}$ - query processing time between nodes $\mathrm{i}$ and $\mathrm{j}$.

Where: $\mathrm{f}_{\mathrm{f}}(z)=P_{p} \cdot P_{f}{ }^{4} \cdot \mathrm{z}^{\mathrm{T}_{12}+T_{23}+T_{34}+T_{45}+T_{56}}$.

Then the average request processing time, provided that

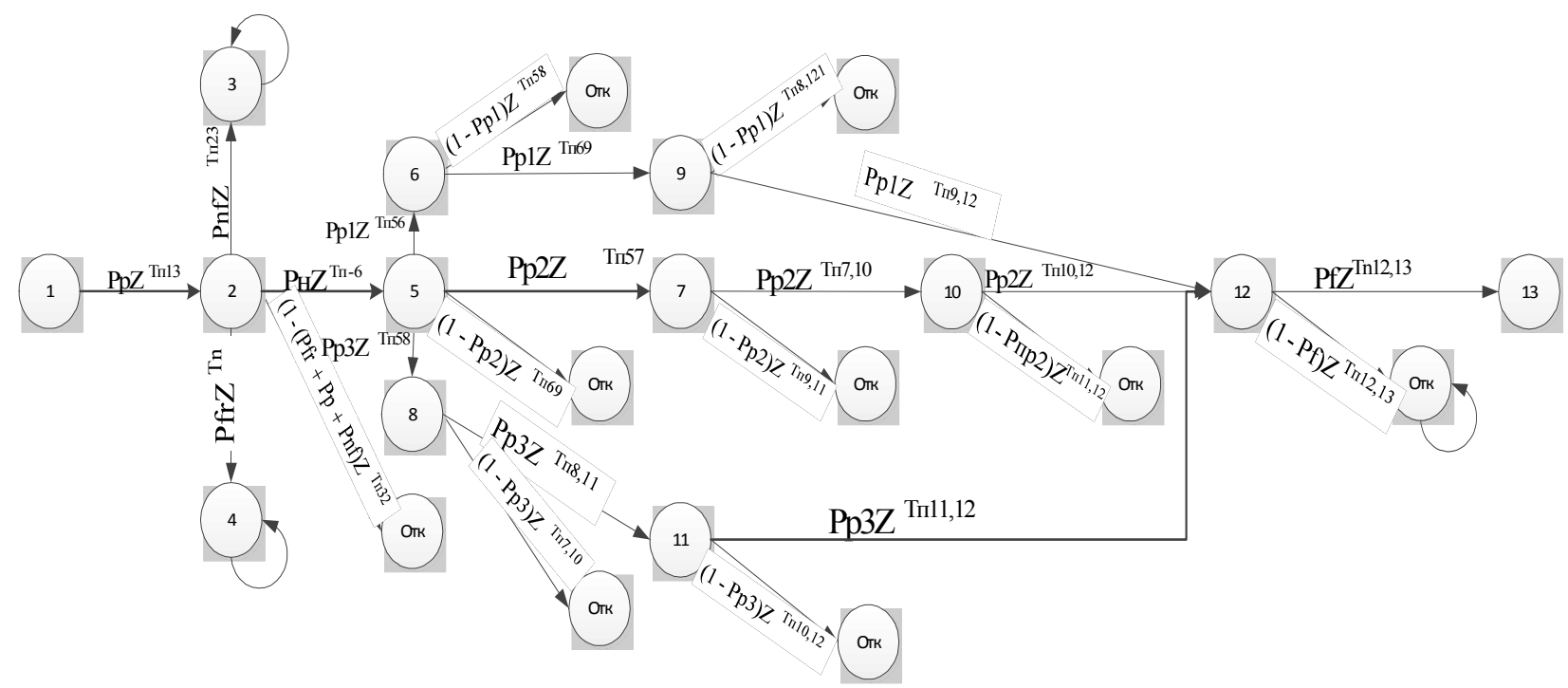

Figure 9. Probable and temporal processing graph of request 
The end view of Probability-time graph is presented in Fig.10.

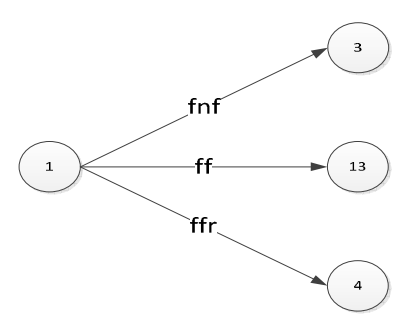

Figure 10. The final form of the transformed graph

The time of servicing a request, provided that the requested service is on the same server is:

$$
\begin{gathered}
T_{s r}=\frac{\mathrm{df}_{\mathrm{p} 0}(\mathrm{z})}{\mathrm{dz}} \mid z=1 \\
T_{s r}=\frac{\mathrm{df}_{\mathrm{f}}(\mathrm{z})}{\mathrm{dz}} \mid(z=1)=P_{p} \cdot P_{f} \cdot P_{f 1} \cdot\left(P_{p 1}^{3}\left(T_{12}+T_{25}+T_{56}+T_{69}+T_{9,12}+T_{12,13}\right)+P_{p 2}^{3}\left(T_{12}+T_{25}+T_{57}+\right.\right. \\
\left.+T_{710}+T_{10,12}+T_{12,13}\right)+ \\
\left.+P_{p 3}^{3}\left(T_{12}+T_{25}+T_{58}+T_{8,11}+T_{11,12}+T_{12,13}\right)\right)+Z^{T_{12}+T_{25}+T_{56}+T_{69}+T_{9,12}+T_{12,13}}+ \\
+Z^{T_{12}+T_{25}+T_{57}+T_{710}+T_{10,12}+T_{12,13}}+Z^{T_{12}+T_{25}+T_{58}+T_{8,11}+T_{11,12}+T_{12,13}}
\end{gathered}
$$

\section{Results}

Graphic representation of the average response time of the load factor for the management multiservice networks (CORBA, SOA, WEB) based on probability-time graphs and generating functions $(7,10)$ is shown in Fig. 11 .

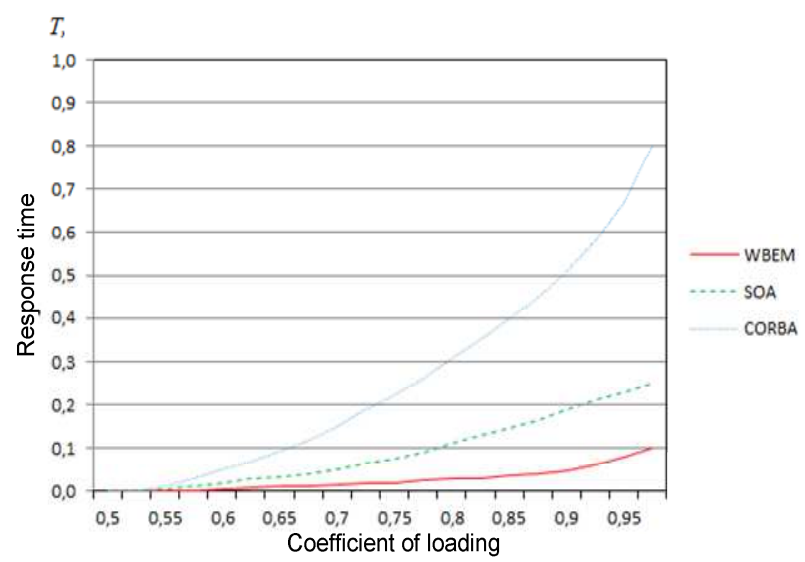

Figure 11. The diagram of depending on the network response time of the load factor

Analysis of dependency showed that the proposed method has reduced the response time (RTT) of 2,5 times. The advantage of the proposed method of managing user requests in multiservice networks based on WBEM for large values of load (load factor network $>0,5$ ) provides features for constructing architectures .

In the network management system based on CORBA, after finding the requested service ORB redirects the re- quest to the appropriate server serving. While the rest of the flow of requests in the queue for service.

Graphic representation of change of average time of request processing with change of intensity of an entering flow for considered architecture is shown in Fig. 12.

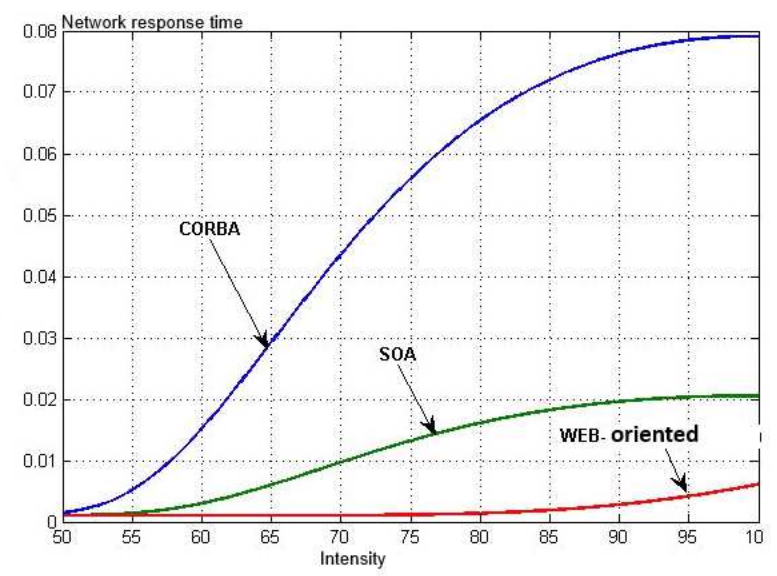

Figure 12. The diagram of dependence of time of response of a network from intensity of arriving requests

In the network management system based on SOA after the repository to locate services, ESB forwards the request to the selected server.

\section{Conclusion}

The analysis of the received dependences showed that average time of response of a network increases with increase in intensity of an entering flow. In the analysis of 
results that fact is confirmed that in case of value of intensity of an entering flow of requests bigger than intensity of service, increase in time of response of a network is much faster for architecture of CORBA, than for SOA and web-centric architecture. This is ensured by features of building architectures. In CORBA, after finding the requested service ORB, redirects the request to the appropriate server service. At the same time the rest of the flow of requests is in the queue for service. In SOA, after the repository has identified the location of service, ESB forwards the request to the selected server. In the offered web-centric architecture the scoring is reached at the expense of multisequencing of process of service of requests on some, in this case three, service servers. In case of arrival of request it redirected on the appropriate proxy-server, that is controlling server group, which provide the type of requested service. The Proxy-server connects to the server which is most suitable for processing of request, and receives an appropriate resource. Thanks to the parallelized processing of requests the architecture allows to service bigger quantity of requests, is steady against failures on separate sections that, in turn, ensures stable functioning of all system at the moments of overloads. As such architecture is rather simply scalable as introduction of new services doesn't affect remaining parts of system.

\section{References}

[1] ITU-T Recommendation M.3010. Principles for a telecommunications management network. The Standish Group. The Scope of Software Development Project Failures: The Standish Group. Stanford, 2009, http://www.cs.nmt.edu/ cs328/reading/Standish.pdf.

[2] E. Ramollari, D. Dranidis, and A. J. H. Simons, A survey of service oriented development methodologies. In: Proceedings of the 2nd European Young Researchers Workshop on Service Oriented Computing, Leicester, UK, June 2007.

[3] Finkel, F. Laroussinie, A. Petit, L. Petrucci, and P. Schnoebelen, Systems and Software Verification: Model-Checking Techniques and Tools, 2009. ISBN 3-540-41523-8

[4] Common Object Request Broker Architecture (CORBA) Specification, Version 3.1 Part 1: CORBA Interfaces

[5] Nicloai M. Josuttis.SOA in Practice. O'Reilly, 2010.

[6] Web-Based Enterprise Management: http://www.dmtf.org/standards/wbem.

[7] David A. Chappell Enterpsice Service Bus. - USA: O’Reilly \& Associates, Inc., 2004. - 27 p.

[8] THOMAS ERL. Introducing SOA Design Patterns. SOA World Magazine, june 2008, Volume 8, issue 6: www.SOA.sys-con.com 